\title{
Visualización de diagramas en el tiempo para sistemas de co- municación digitales basados en hardware reconfigurable
}

\author{
Melissa Eugenia Diago ${ }^{1}$ y William Augusto Gómez ${ }^{1}$
}

\section{Resumen}

Debido a la escases de herramientas software capaces de desplegar diagramas en el tiempo, se concibe el proyecto de visualización de diagramas en el tiempo para sistemas de comunicación digitales basados en hardware reconfigurable, en el cual, a partir de una serie de modulaciones implementadas en la tarjeta SPARTAN-3A busca generar una interfaz gráfica capaz de mostrar dichos diagramas. Por lo que inicialmente se detallan los conceptos básicos de este tipo de FPGA y se realiza una introducción a los diagramas en el tiempo, para proseguir con el desarrollo de la interfaz gráfica mediante el entorno de desarrollo GUIDE de MATLAB, en donde a través de diversas ventanas se logra la recepción, procesamiento y visualización de la información proveniente del computador. Adicionalmente se muestra la implementación de la interfaz para cada modulación desplegando los diagramas en el tiempo para los datos correspondientes a antes y después del canal AWGN. Finalmente se tiene el análisis de los resultados obtenidos, así como la validación de estos a partir de los diagramas generados por Simulink.

\section{Abstract}

The current lack of software capable of displaying the different ways of looking at a digitally-modulated signal in time produces the idea of creating a Graphical User Interface (GUI) that takes a number of digital modulation schemes implemented on a FPGA SPARTAN-3A and plot the eye diagram, constellation diagram, and signal trajectory. This GUI is designed and implemented using MATLAB's GUI Development Environment (GUIDE), which provides a tool for designing user interfaces for custom applications. In this paper, the analysis and results of each diagram obtained with this GUI are presented. Comparisons with diagrams obtained with Simulink are as well included.

Índice de Términos-Diagramas en el tiempo, interfaz gráfica de usuario, modulaciones digitales, SPARTAN-3A.

1 Universidad del Cauca, Colombia 



\section{Herramienta hardware}

Un arreglo de compuertas lógicas programables (FPGA, Field Programmable Gate Array) es un dispositivo semiconductor que contiene bloques de lógica reprogramable dispuestos para la implementación de funcionalidades personalizadas en hardware, desarrolladas en tareas de cómputo digital en software y compiladas en un archivo de configuración (bitstream) que contiene información acerca de las conexiones que deben ser realizadas internamente. Asimismo, estos dispositivos son reconfigurables por lo que toman una nueva lógica cuando se compilan con una configuración diferente, también poseen un buen rendimiento que permite maximizar cada ciclo de reloj, además sobresalen sus precios factibles y su fiabilidad se presenta como ventaja fundamental al momento de poner en ejecución un proyecto, ya que mientras las herramientas software ofrecen un entorno de programación, los circuitos de un FPGA son una implementación segura de la puesta en marcha de un programa.

\section{Diagramas en el tiempo}

Para el análisis del comportamiento de los sistemas de comunicaciones digitales se emplean diversas técnicas y mediciones de desempeño que permiten lograr una apreciación de la calidad del enlace de transmisión. Es por esto que los diagramas en el tiempo son primordiales para el análisis de la señal, de sus formas de onda y sus símbolos propagados en un enlace de comunicaciones, logrando la visualización de los diversos mecanismos de degradación, niveles de ruido, potencia de la señal entre otros.

Es así como el diagrama de constelación, la trayectoria de la señal y el diagrama del ojo permiten examinar la señal de transmisión a lo largo del enlace de comunicaciones.

\section{A. Diagrama de Constelación}

Es un método de representación de los estados de los símbolos en el plano complejo en términos de amplitud y fase, las componentes de los símbolos que se encuentran en fase con la señal portadora hacen referencia al eje horizontal y las componentes en cuadratura (90) al eje vertical (Packard, 1997). 


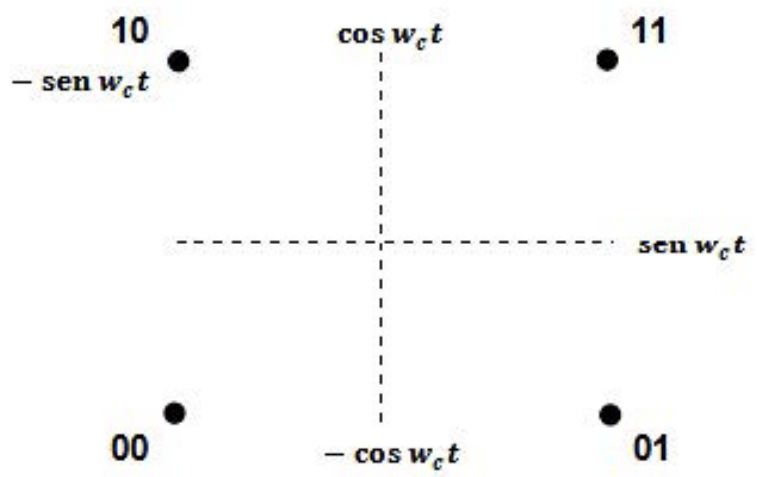

Figura 1. Diagrama de constelación para QPSK.

Básicamente la constelación de la Fig. 1 ilustra el modulo que está dado por la distancia que separa el origen de las coordenadas y la ubicación del símbolo, y la fase que corresponde al ángulo que se forma con el eje horizontal de cada posible símbolo que conforma la modulación.

\section{B. Trayectoria de la Señal}

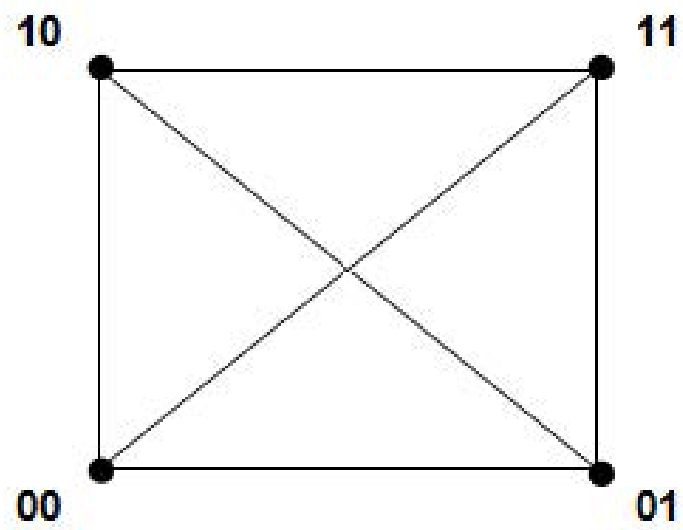

Fig. 2. Trayectoria de la señal para QPSK.

Este tipo de diagrama permite visualizar los caminos de las transiciones entre todos los posibles estados de una señal modulada (The MathWorks I. , DiscreteTime Signal Trajectory Scope, 1994), debido a que grafica la componente de la señal en fase en función de la componente en cuadratura.

A través del diagrama de la Fig. 2 se observan puntos de convergencia, que equivalen a los símbolos representados en el diagrama de la Fig. 1. 


\section{Diagrama del Ojo}

La calidad de una señal digital y la integridad del sistema que lo transmite pueden ser medidas con satisfacción a través del diagrama del ojo. Este diagrama corresponde a la superposición de las combinaciones de los posibles unos y ceros en un rango de tiempo o cantidad de bits de la señal determinada.

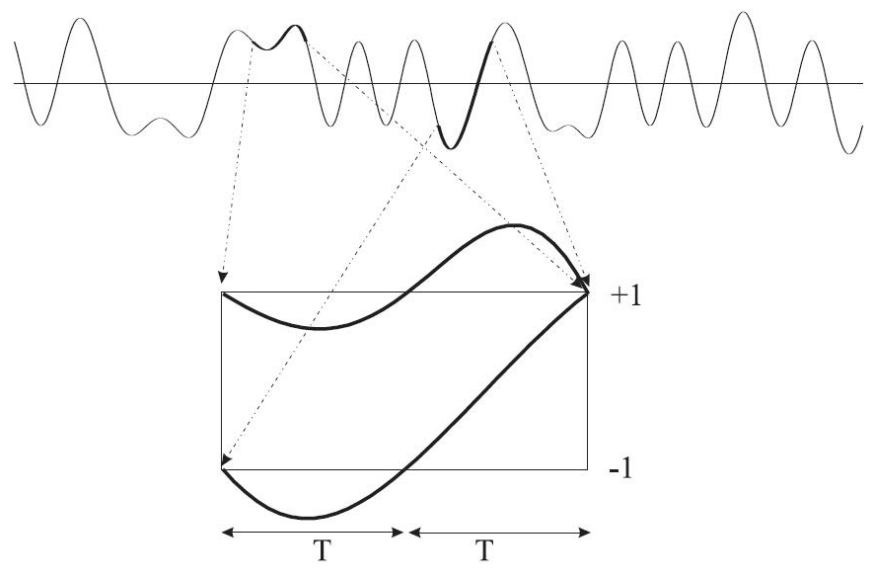

Fig. 3. Proceso de construcción de un diagrama de ojo.

La Fig. 3 ilustra la formación del diagrama de ojo. El llamado ojo aparece en el centro de la figura como la zona vacía, en ausencia de ruido o interferencia entre símbolos todos los trazos pasan por los mismos puntos en el centro, que se corresponden con los niveles de la señal utilizada. Al muestrear justamente en los instantes que se corresponden con dichos puntos, se obtendría el símbolo enviado en dichos instantes (Artés Rodríguez, y otros, 2012). La apertura vertical del ojo es una buena medida de la inmunidad al ruido, puesto que da una idea de qué amplitud de ruido sería necesaria para producir un error y la apertura horizontal, da una medida de la inmunidad a los errores en el instante elegido para muestrear la señal.

\section{Desarrollo de la interfaz grafica}

Una interfaz gráfica de usuario (GUI, Graphical User Interface) hace referencia a un software que funciona como puente de comunicación entre una computadora y su usuario, permitiéndole interactuar con esta a través del uso de símbolos, metáforas visuales y la opción de seleccionar y arrastrar elementos con el mouse en vez de introducir texto como comandos de línea. Los sistemas que funcionan junto a una GUI son más fáciles y simples de entender y manejar, esto debido a que el usuario no necesita conocer, memorizar o aprender comandos nuevos o adicionales, razones 
por las cuales hoy en día las interfaces graficas de usuario son empleadas por la mayoría de los sistemas operativos modernos.

A continuación se detalla cada etapa del diseño establecido para el desarrollo de la interfaz gráfica, el cual se muestra en la Fig. 4.

FPGA SPARTAN 3A

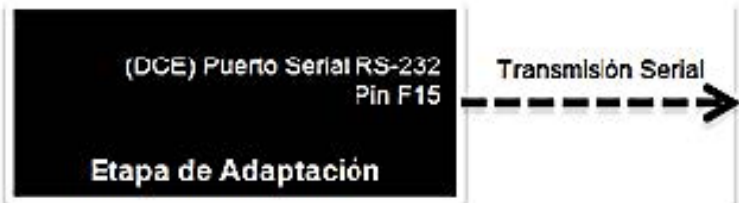

INTERFAZ GRAFICA

\section{Puerto USB}

Etapa de Visualización

Fig. 4. Diagrama de bloques general del sistema.

\section{A. Adaptación a la interfaz gráfica}

El trabajo de grado desarrollado tiene como objetivo visualizar los diagramas en el tiempo de las señales generadas por el modulador y el canal AWGN de los diferentes proyectos planteados en (Quintero \& Jojoa, 2013) por lo cual se hace indispensable la adaptación de estos datos a un formato que permita ser enviado a través del puerto serial RS-232 que tiene un único pin asignado para la transmisión de datos.

En la Fig. 5 se observa el modelo diseñado en MATLAB R2012a en la herramienta Simulink con Xilinx System Generator, con el cual se realiza el respectivo tratamiento que se le debe hacer a los datos para poder transmitirlos hacia el PC, así como también se visualizan los formatos de las palabras binarias con las que se trabaja. En esta se visualizan dos bloques pequeños que se refieren a la modulación y el canal AWGN diseñados por los otros proyectos. El bloque de mayor tamaño representa el subsistema que conforma el diseño de la etapa de adaptación ejecutada, que específicamente implementa una lógica combinacional encargada de generar un protocolo de transmisión específico, de tal manera que se maneje el mismo lenguaje en el extremo transmisor y receptor.

La adaptación diseñada se conforma por un conjunto de bloques de la librería de Xilinx, que se encargan inicialmente de registrar una cantidad específica de los datos provenientes de los bloques anteriores para luego ser ordenados en un vector conformado por tres banderas que indican el inicio de los datos modulados, el paso a datos modulados contaminados con ruido y el fin de la trama generada. Para luego ser convertidos al formato UFix_1_0 que indica que los datos solo se conforman por un bit, esto es necesario para serializar la salida de información. Finalmente todo el sistema se encuentra controlado por una "tasa de muestreo" 
que se encarga de poner en la salida un bit cada 5208 tiempos, que se refieren a la cantidad de tiempos que tienen que pasar para obtener una velocidad de transmisión de $9600 \mathrm{bps}$ a partir de los 50MHz del FPGA seleccionado.

Esta última tarea de sincronización es fundamental para lograr la recepción de los datos sin pérdida alguna.

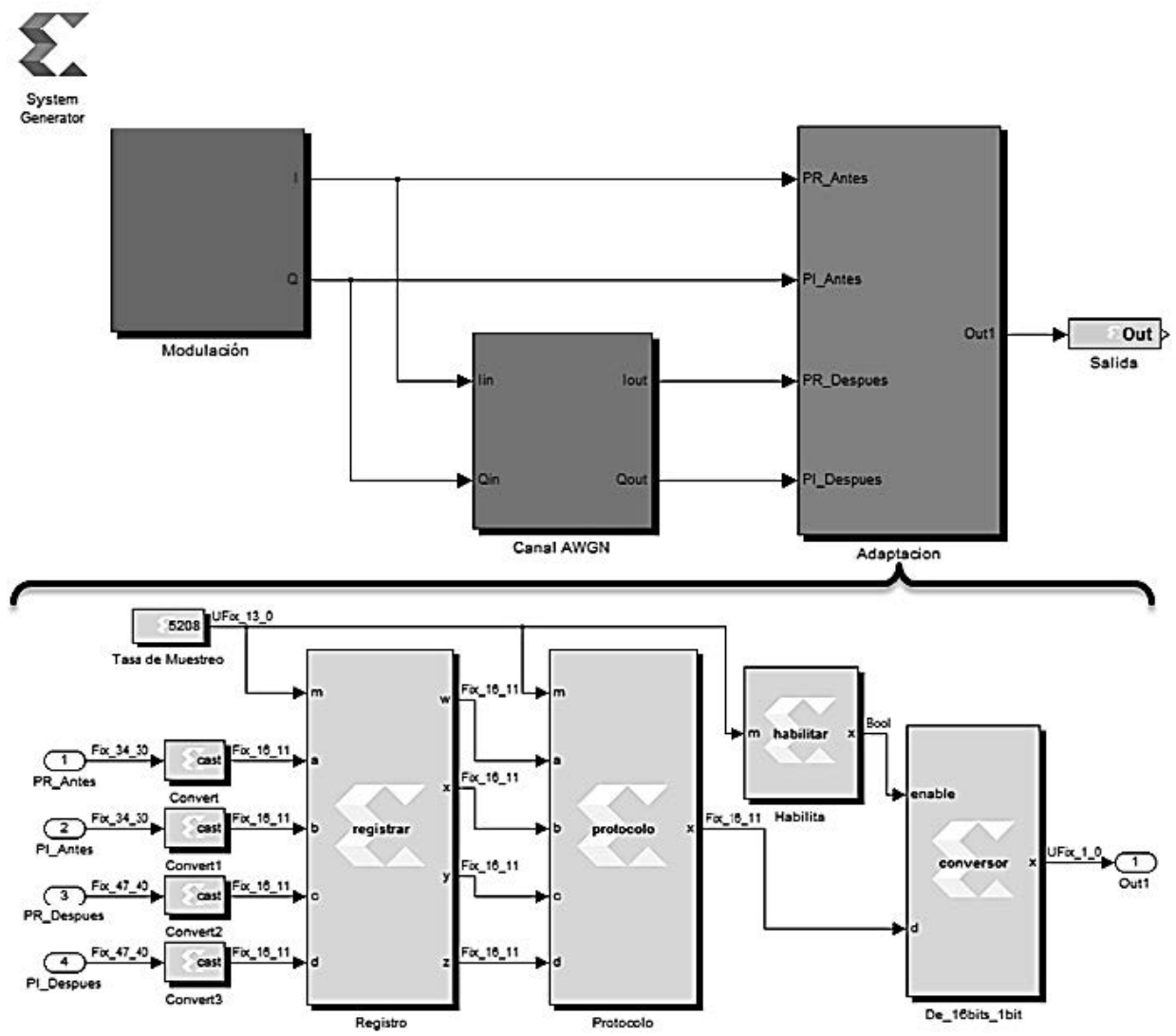

Fig. 5. Modelo en Simulink con SysGen para la adaptación de los datos.

\section{B. Diseño y visualización de la interfaz gráfica}

A continuación se detalla la realización de la interfaz gráfica, la cual se desarrolla con el objetivo de entablar una comunicación entre el usuario y el equipo, simplificando su uso al momento de ser ejecutada y cuando se inicia el proceso de recepción de datos provenientes del FPGA a través del puerto RS-232. Mediante un diseño fácil de accionar, el usuario contara con botones y ventanas con las cuales podrá visualizar los diagramas del tiempo antes y después del canal. 


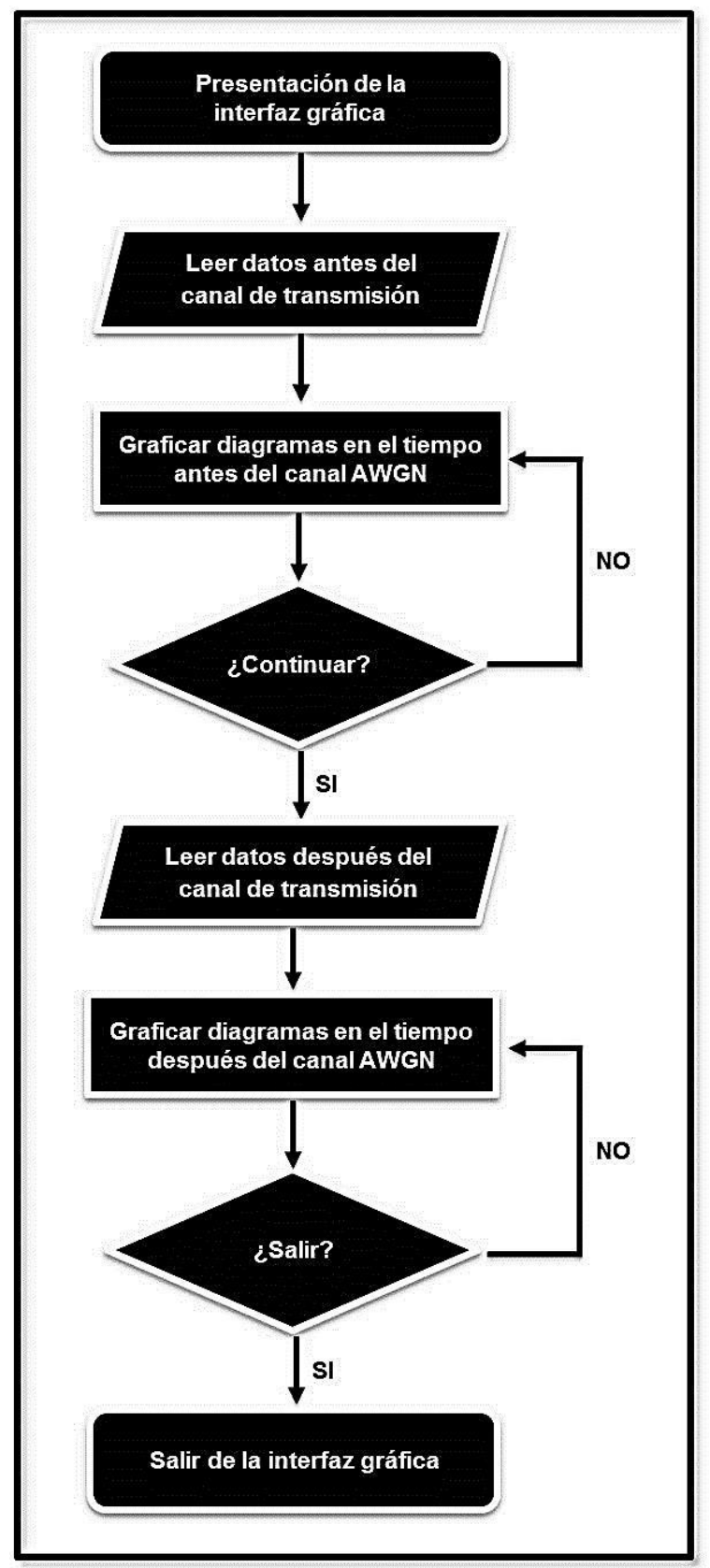

Fig. 6. Diagrama de flujo de la interfaz gráfica.

Las interconexiones entre cada ventana que conforman la interfaz gráfica, así como las acciones que se ejecutan en cada una de estas, se especifican en el diagrama de flujo de la Fig. 6. 


\section{1) Presentación de la interfaz gráfica}

La ventana principal de la interfaz gráfica es la que se muestra en la Fig. 7, en esta se encuentra la presentación tanto del proyecto como la de los encargados de este, además del botón iniciar el cual al ser presionado inicia la lectura de los datos y despliega la ventana de diagramas en el tiempo antes del canal, almacenando los datos de la primera trama correspondientes a antes y después del canal.

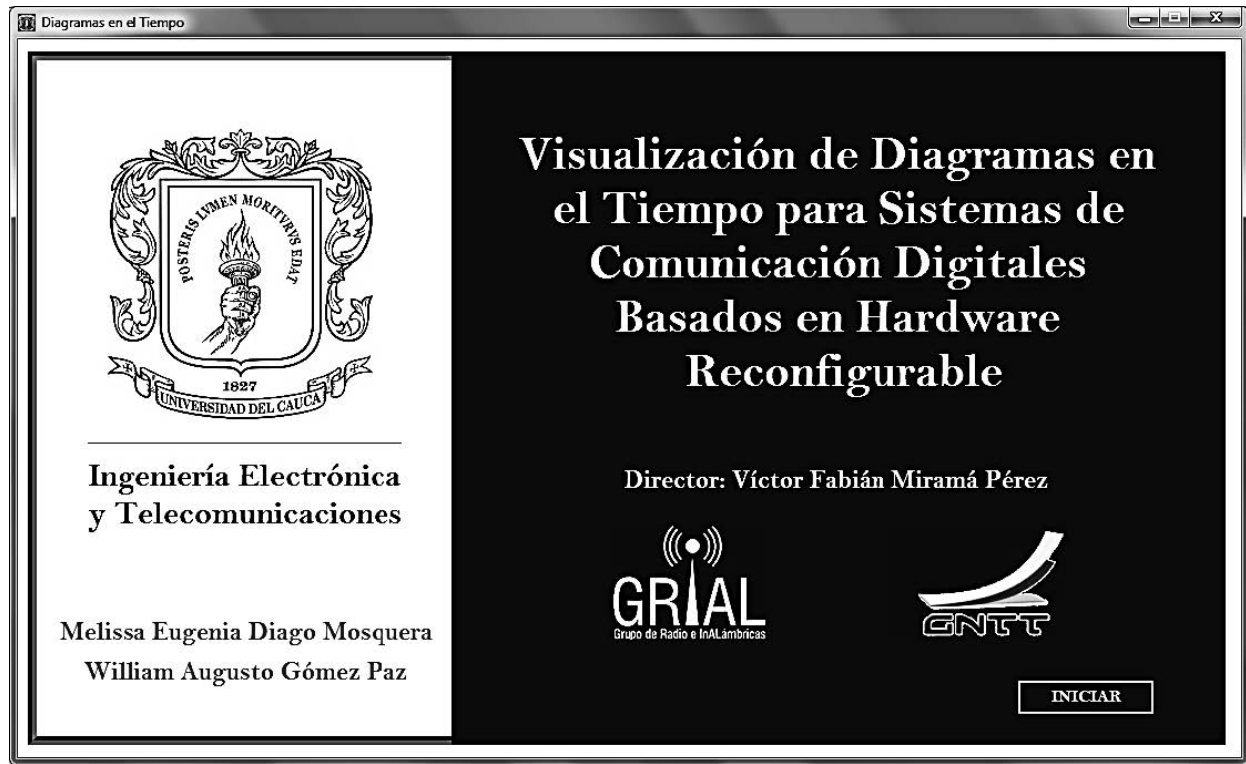

Fig. 7. Presentación de la interfaz gráfica.

\section{2) Graficar diagramas en el tiempo}

Para lograr la visualización de los diagramas en el tiempo antes y después del canal de transmisión fue necesaria la creación de dos interfaces de usuario, cada una cuenta con 4 espacios dispuestos para graficar el diagrama de constelación, la trayectoria de la señal y el diagrama del ojo en fase y cuadratura, además de esto contienen un botón para dar paso al siguiente proceso.

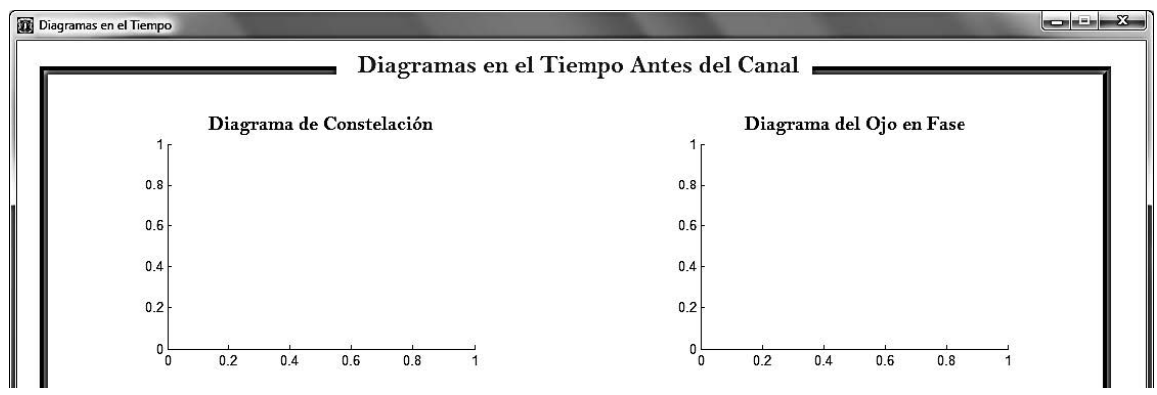




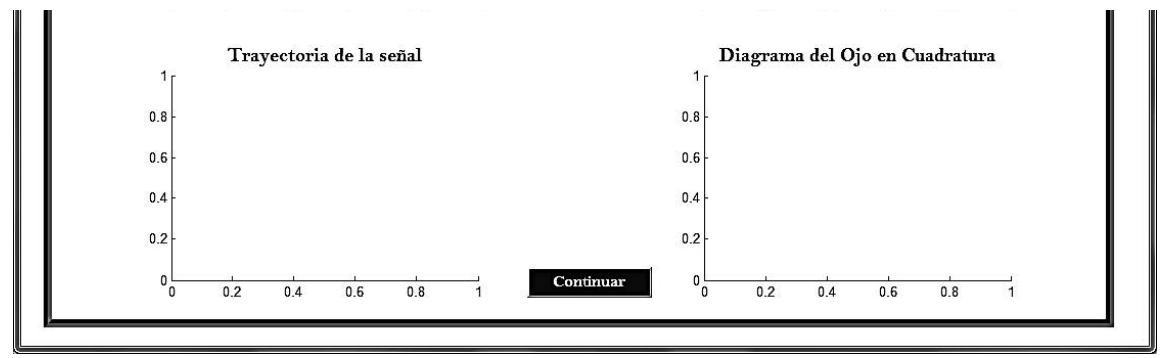

Fig. 8. Visualización de diagramas en el tiempo antes del canal.

En la Fig. 8 se observa la interfaz de usuario que despliega los diagramas en el tiempo antes del canal; en el momento que sucede el opening de esta interfaz se observan todos los diagramas en el tiempo de la primera trama de datos recibidos antes del canal que han sido almacenados previamente en el opening de la presentación de la interfaz gráfica, al pasar 0,1 segundos la interfaz se refresca y grafica otra trama de datos con el fin de actualizar la información y lograr una aproximación de lo que sucede en tiempo real.

Al oprimir el botón "Continuar" se da lugar al opening de la interfaz de usuario de la Fig. 9 en la cual se repite el proceso de la anterior ventana, con la diferencia de que los datos a graficar corresponden a la señal después del canal de transmisión, visualizando diagramas con ruido, puesto que el canal AWGN que se utiliza para transmitir la señal se lo agrega. El botón "Desconectar" finaliza el proceso y cierra la interfaz de usuario dando por concluida la visualización de los diagramas en el tiempo de la señal modulada.

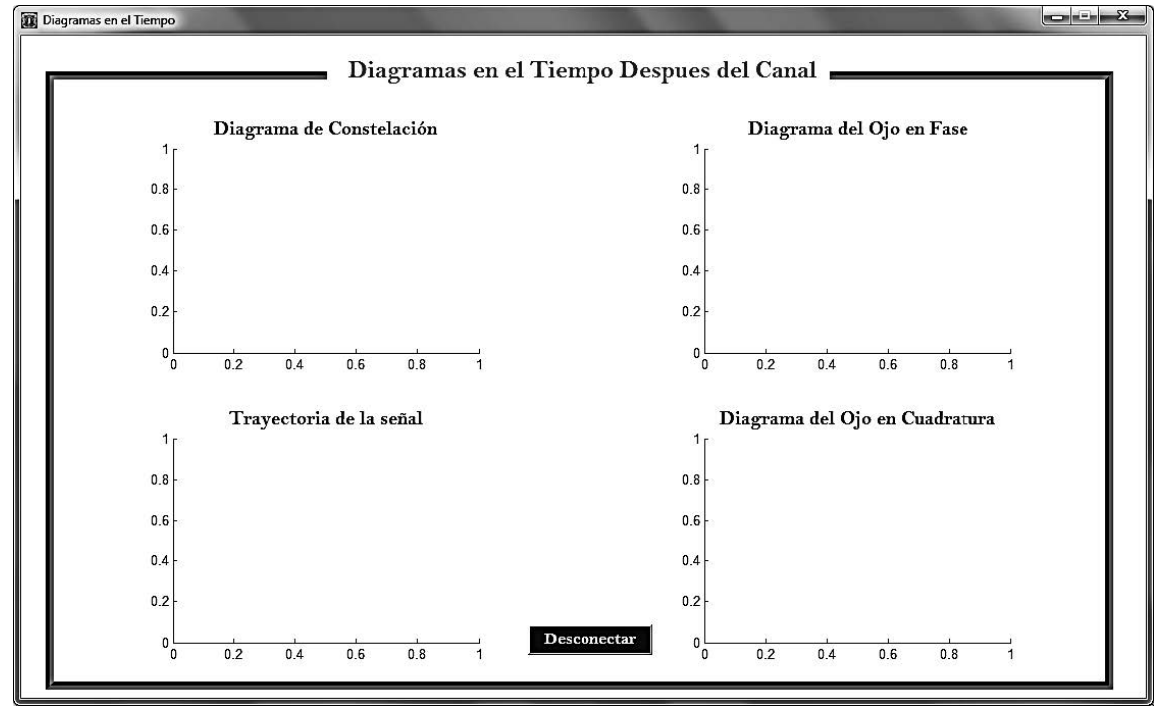

Fig. 9. Visualización de diagramas en el tiempo después del canal. 


\section{Resultados}

Al concluir el diseño, desarrollo e implementación de la interfaz gráfica se hace necesario validar los resultados obtenidos antes y después del canal a partir de los diagramas generados por los bloques Discrete-Time Scatter Plot Scope ${ }^{2}$ (The MathWorks I. , 1994), Discrete-Time Signal trajectory Scope ${ }^{3}$ (The MathWorks I. , Discrete-Time Signal Trajectory Scope, 1994) y Discrete-Time Eye Diagram Scope ${ }^{4}$ (MathWorks, Discrete-Time Eye Diagram Scope, 1994) de Simulink.

Para el análisis de resultados en el actual artículo, se toma como ejemplo el modulador por desplazamiento de fase en cuadratura (QPSK, Quadrature Phase Shift Keying) y su respectivo canal AWGN desarrollados por el proyecto "Análisis del desempeño de un sistema de comunicaciones con modulaciones BPSK, QPSK, 8PSK y OQPSK basado en hardware reconfigurable" del macro proyecto mencionado en (Quintero \& Jojoa, 2013).

A. Simulaciones a partir de los bloques de Simulink

El montaje general para desplegar los diagramas en el tiempo a través de los bloques suministrados por la biblioteca de Simulink se observa en la Fig. 10, la cual cuenta con un bloque azul que se refiere a la modulación específica a visualizar, seguido de un bloque rojo que contiene el canal AWGN, finalmente se encuentran los bloques encargados de graficar los diagramas en el tiempo antes y después del canal, resaltados con color verde.

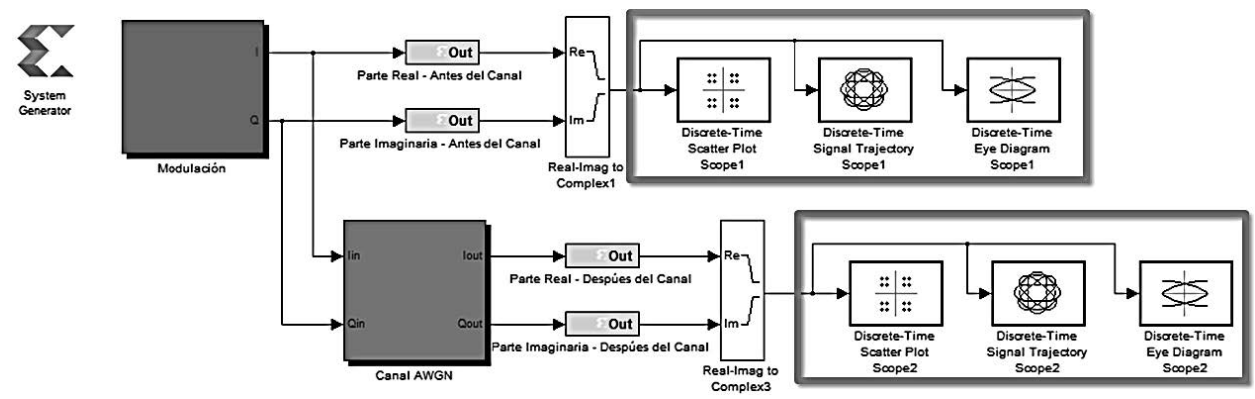

Fig. 10. Bloques de Simulink para graficar diagramas en el tiempo.

A continuación se visualizan los diagramas en el tiempo generados antes y después del canal AWGN de la modulación seleccionada, realizando dos variaciones de Eb/No para observar los cambios que ocurren en los diagramas después del canal.

\footnotetext{
Muestra el diagrama de constelación de una señal modulada.

Muestra la trayectoria de la señal modulada.

4 Muestra el diagrama del ojo en fase y cuadratura de la señal modulada.
} 


\section{1) Antes del canal}

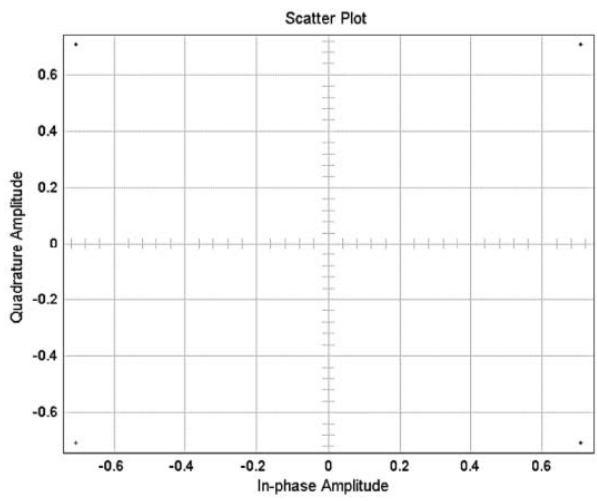

(a)

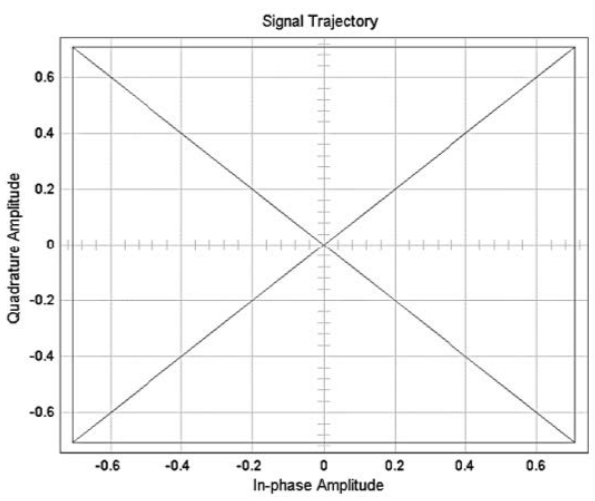

(b)
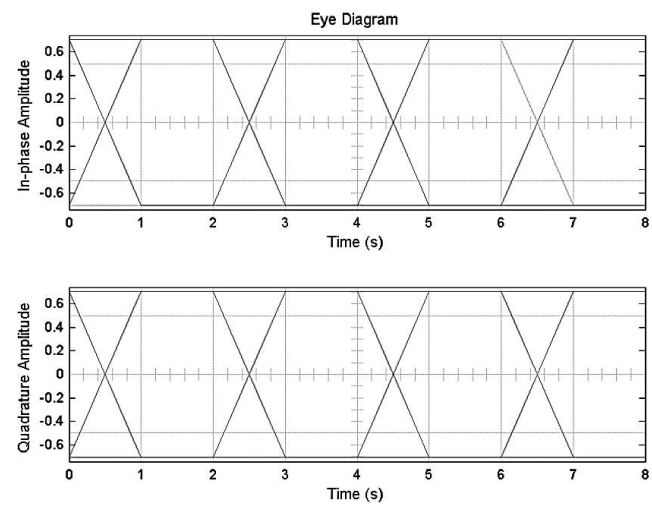

(c)

Fig. 11. (a) Diagrama de constelación. (b) Trayectoria de la señal. (c) Diagrama d el ojo. Modulación QPSK.

La Fig. 11 muestra los diagramas en el tiempo generados por los bloques de Simulink para el modulador. Validando el diagrama de constelación teórico del modulador QPSK de Simulink (MathWorks, QPSK Modulator Baseband, 1994) con el de la Fig. 11 (a), se observa la correcta formación del diagrama de constelación, al igual que la trayectoria de la señal (Fig. 11 (b)). Con respecto al diagrama del ojo se comprueba la superposición de los cambios de la señal en un periodo de tiempo, observándose claramente el instante óptimo de muestreo en el eje x y el de decisión en el eje y. 


\section{2) Después del canal}

La Fig. 12 muestra los diagramas en el tiempo generados por Simulink para el modulador QPSK con el parámetro Eb/No del canal AWGN configurado a 14.
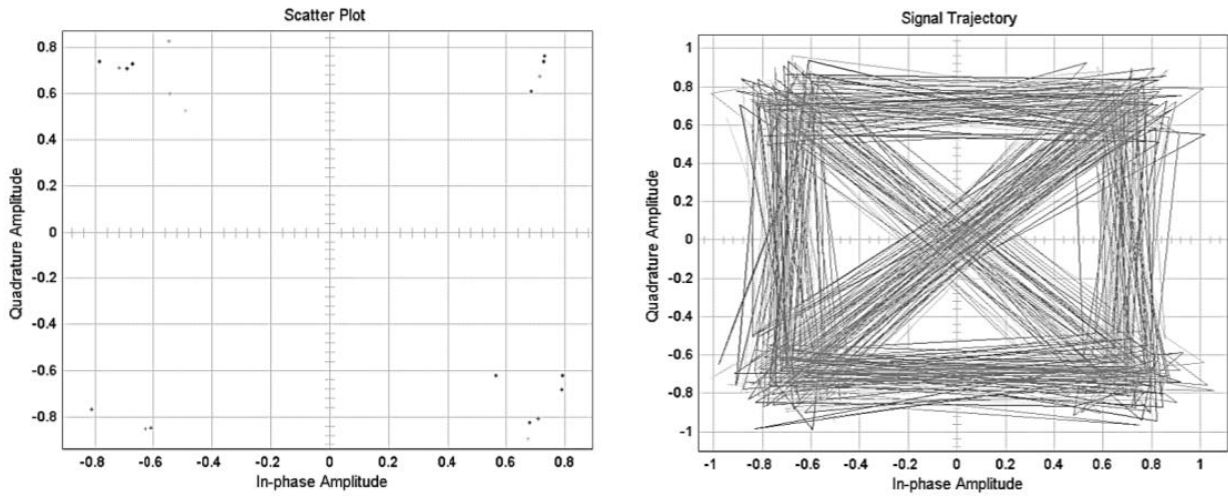

(a)

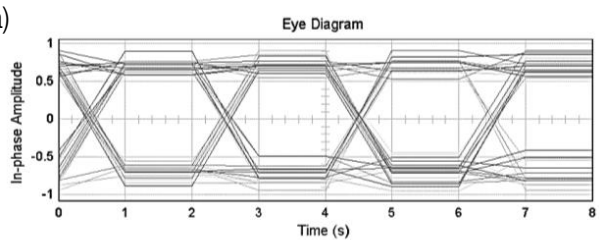

(b)

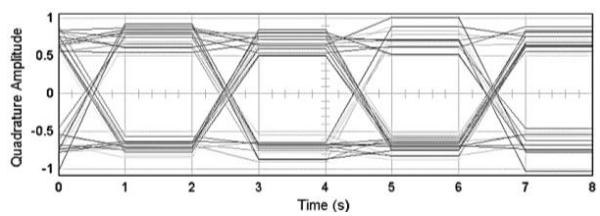

(c)

Fig. 12. (a) Diagrama de constelación. (b) Trayectoria de la señal. (c) Diagrama del ojo. Modulación QPSK. Modulación QPSK con Eb/No = 14 .

La Fig. 13 muestra los diagramas en el tiempo generados por Simulink para el modulador QPSK con el parámetro Eb/No del canal AWGN fijando en 21.

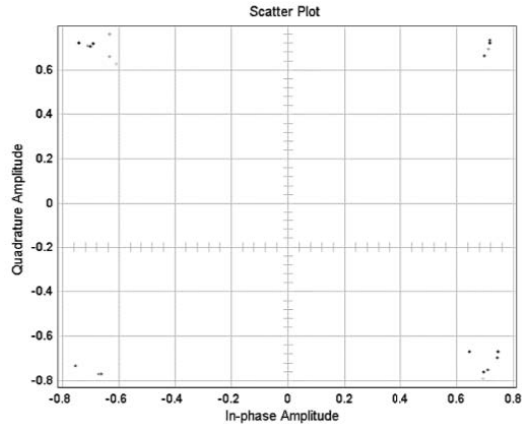

(a)

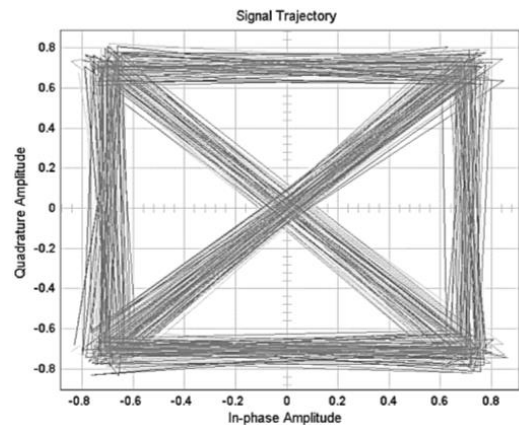

(b) 

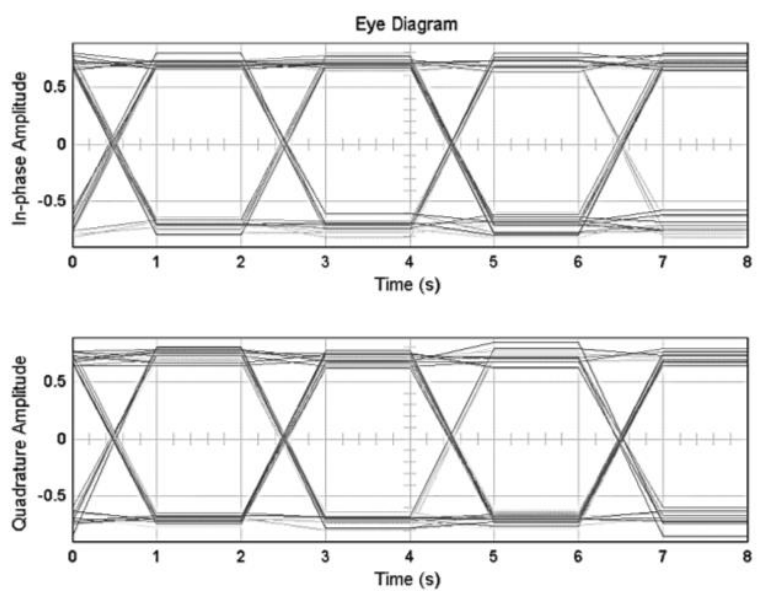

(c)

Fig. 13. (a) Diagrama de constelación. (b) Trayectoria de la señal. (c) Diagrama del ojo. Modulación QPSK. Modulación QPSK con Eb/No $=21$.

Al observar los diagramas generados después del canal se evidencia la presencia del ruido, degradando claramente los diagramas. En el caso del diagrama de constelación los puntos se dispersan alrededor del estado inicial, el trazo de la trayectoria de la señal deja de ser una línea entre cambios de estado y el diagrama del ojo presenta continuas variaciones al ser analizado en un tiempo específico.

\section{B. Simulaciones a partir de la interfaz gráfica diseñada}

Mediante la configuración realizada en Simulink System Generator se obtienen los datos necesarios para que la interfaz gráfica despliegue los diagramas en el tiempo antes y después del canal, en la Fig. 14 se muestra el diagrama de bloques que surge de esta comunicación.

FPGA SPARTAN 3A

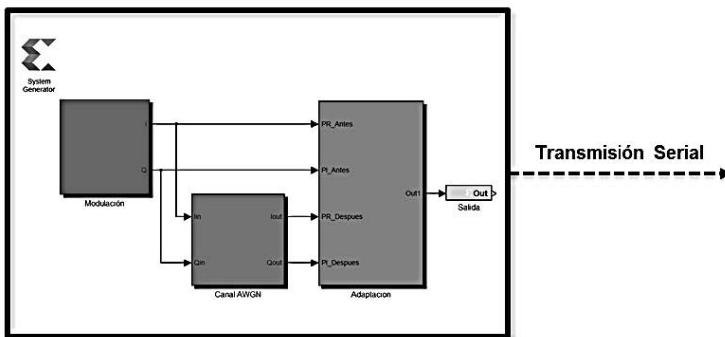

INTERFAZ GRAFICA

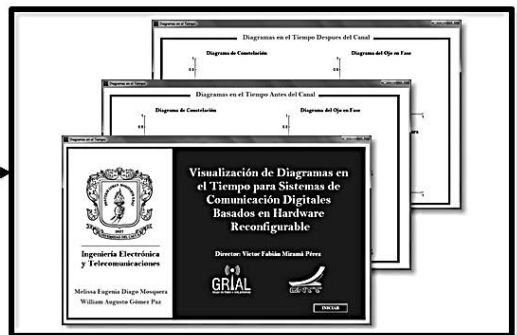

Fig. 14. Diagrama de bloques para la visualización de diagramas en el tiempo a través de la interfaz gráfica. 


\section{1) Antes del canal}

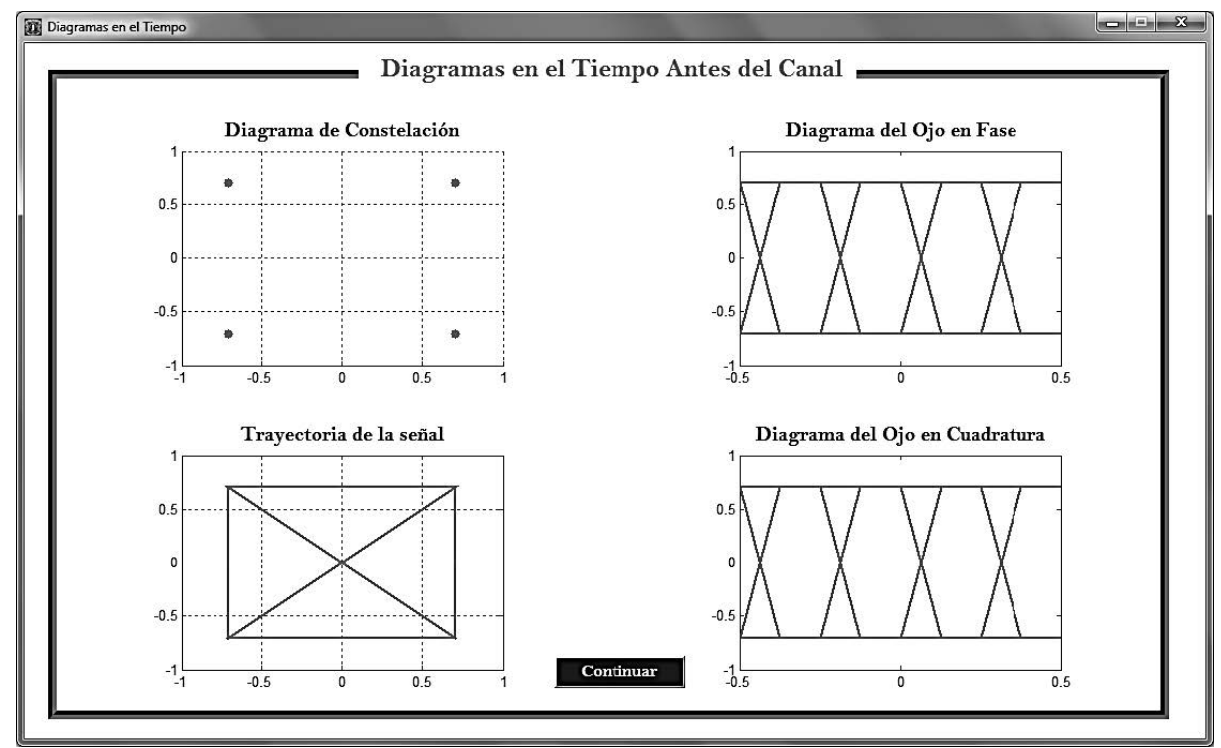

Fig. 15. Diagramas en el tiempo generados por la interfaz gráfica para QPSK.

La Fig. 15 contiene los diagramas en el tiempo generados por la interfaz gráfica para el modulador QPSK. En los cuales el diagrama de constelación visualiza los cuatro estados equidistantes que representan a la modulación sin ninguna presencia de dispersión, presumiéndose ausencia total de ruido, en cuanto a la trayectoria de la señal, se aprecia como todos los trazos entre estados convergen a un solo punto, evidenciando la representación de los símbolos, corroborándose toda esta información a partir de la Fig. 12 (a y b). Con respecto al diagrama del ojo en fase y cuadratura, son semejantes a los de la Fig. 12 (c) puesto que conservan la forma de la señal generada, a pesar de ser graficados en periodos de tiempos diferentes, además de esto se evidencian los dos posibles valores que puede tomar la señal modulada tanto en el eje real como en el eje imaginario. 


\section{2) Después del canal}

Para visualizar los cambios que pueden ocurrir después del canal debido al ruido blanco gaussiano aditivo (AWGN) se realizan dos variaciones de Eb/No.

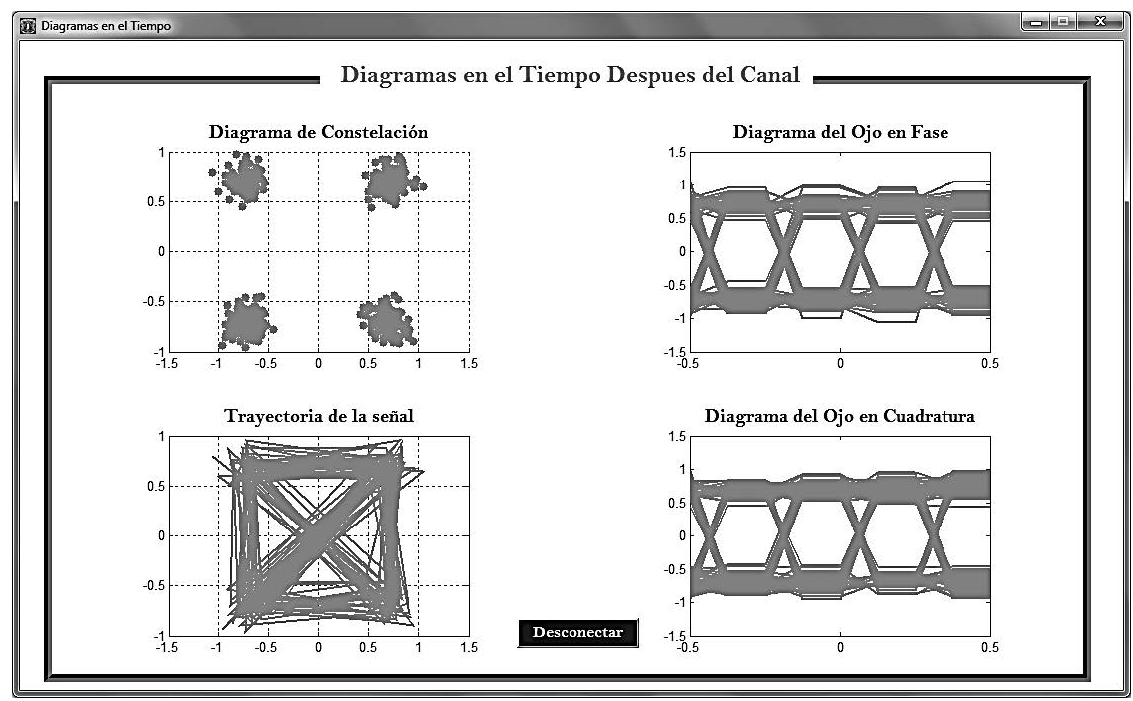

Fig. 16. Diagramas en el tiempo generados por la interfaz gráfica para QPSK con Eb/No $=14$.

La Fig. 16 muestra los diagramas en el tiempo generados por la interfaz para la modulación QPSK con un Eb/No de 14 y la Fig. 17 muestra los diagramas en el tiempo generados por la interfaz para la modulación QPSK con un Eb/No de 21.

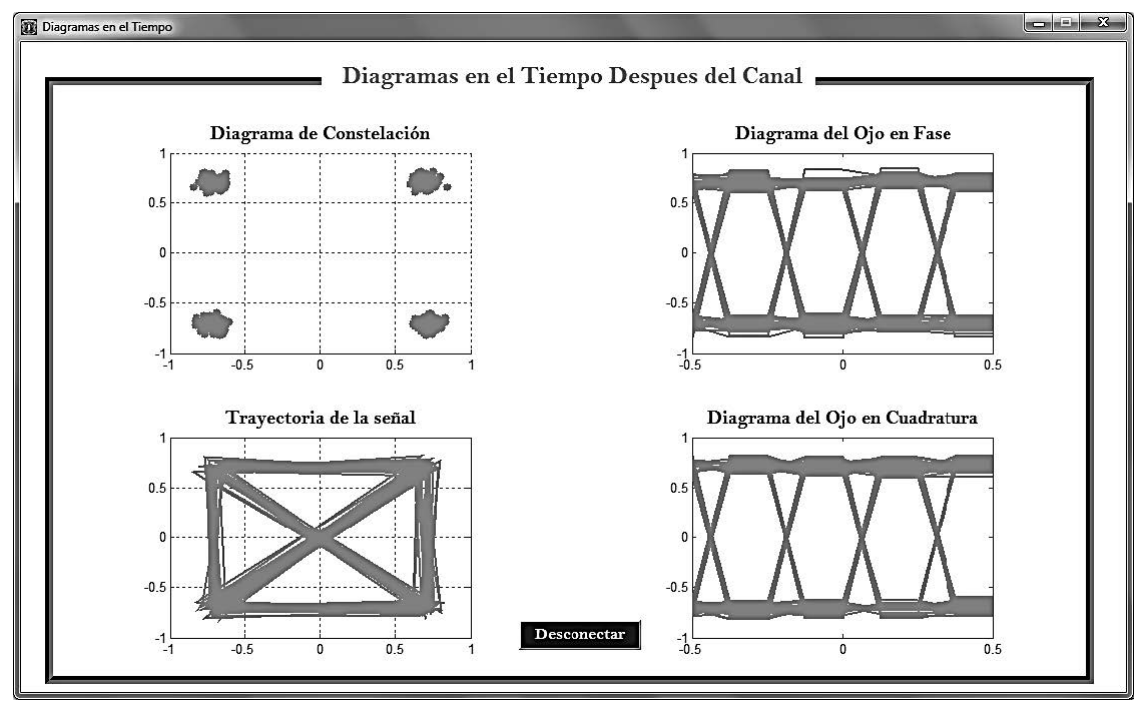

Fig. 17. Diagramas en el tiempo generados por la interfaz gráfica para QPSK con Eb/No $=21$. 
Al obtener las gráficas después del canal con las correspondientes variaciones en el valor de $\mathrm{Eb} / \mathrm{No}$, se demuestra que a través de la interfaz gráfica se puede ver como a un mayor valor de Eb/No las gráficas presentan mayor concentración sobre los puntos generados antes del canal. Verificando el correcto funcionamiento de la herramienta de visualización diseñada con la modulación QPSK.

\section{Conclusiones}

La etapa de adaptación diseñada e implementada en la tarjeta SPARTAN-3A permite la transmisión de los datos modulados antes y después del canal AWGN de una manera ordenada y secuencial siguiendo un protocolo de transmisión delimitado por banderas establecidas en el código incorporado. Haciendo del sistema un conjunto flexible, que permite la adaptación de cualquier formato de dato, presentando como único requerimiento la existencia de dos números complejos representados por su componente real e imaginaria.

A través de la herramienta software desarrollada se proporciona un entorno visual sencillo que logra la interacción entre la tarjeta SPARTAN-3A y el computador mediante el cable convertidor de serial a USB, posibilitando la visualización del diagrama de constelación, la trayectoria de la señal y el diagrama del ojo en fase y cuadratura de los datos modulados antes y después del canal AWGN.

Al analizar y visualizar los resultados de las simulaciones realizadas se concluye que los diagramas en el tiempo son satisfactorios ya que los diagramas en el tiempo desplegados por los bloques de Simulink son similares a los obtenidos por la interfaz gráfica.

Como consecuencia de una recepción de únicamente 500 bits, se hizo necesaria la actualización de estos datos cada 0,1 segundos para proporcionar una mejor formación de cada diagrama y así obtener una visualización en periodos de tiempo real, si se habla de recepción de datos en tiempo real, no se logra en su totalidad puesto que al tener que recargar los datos se pierden periodos de tiempo logrando solo una aproximación a través de muestras del tiempo real.

Pese a la carencia de herramientas que tiene la interfaz gráfica para evaluar el desempeño, ruido o interferencia entre símbolos de cada uno de los diagramas en el tiempo, presenta una fluida visualización de estos lo cual permite al usuario realizar un análisis visual acerca de estos factores y a partir de ahí obtener los resultados necesarios.

Debido a limitaciones en los recursos por parte de la tarjeta SPARTAN-3A, no es posible generar una interfaz gráfica que despliegue las múltiples modulaciones estudiadas en este trabajo de grado, por ende, se debe trabajar con cada una de estas por separado, es decir, que se requiere programar la tarjeta con la modulación que se quiere analizar y posteriormente programar otra si así se desea. 


\section{Recomendaciones}

Se recomienda identificar el tipo de formato que se requiere, ya sea en punto fio, punto flotante o booleano, puesto que una vez esté definido basta con comprobar que se mantenga a medida que se incrementa la lógica del modelo.

Se aconseja verificar previamente si la salida se encuentra realmente serializada o está en paralelo a través de la visualización de su formato.

Se sugiere implementar desde un inicio el protocolo de transmisión RS-232 en el cual se establece un bit de Start fijado en 0 y un bit de Stop fijado en 1 por cada 8 bits transmitidos, para la correcta recepción de los datos.

Para lograr la sincronización entre la transmisión y recepción de datos se recomienda identificar la frecuencia de trabajo de la tarjeta que se esté utilizando y la frecuencia a la que se desea recibir, puesto que a partir de la frecuencia del FPGA se va a obtener la frecuencia de recepción, es decir, en el caso de este trabajo de grado la frecuencia que maneja la tarjeta SPARTAN-3A es de $50 \mathrm{MHz}$, y la de recepción es de $9600 \mathrm{~Hz}$, por lo que al dividir $50 \mathrm{MHz}$ entre los $9600 \mathrm{~Hz}$ se obtiene una razón de 5208, resultado que habilita la salida de datos.

Se sugiere poner todo lo necesario en términos de una sola variable para alcanzar la flexibilidad y adaptabilidad que un sistema requiere.

Es importante utilizar el bloque "Resource Estimator" de la librería de Xilinx en Simulink, ya que permite dar una aproximación del área ocupada en el FPGA, así que si esta excede los límites de recursos brindados por el datasheet de la tarjeta, se hace necesario el cambio a una tarjeta con mayor recursos o en el peor de los casos rediseñar el sistema establecido.

Para las pruebas previas a la recepción final se recomienda hacer uso de programas externos a la interfaz gráfica, ya que mediante la visualización de los datos se puede comprobar si estos corresponden a los transmitidos. Programas como Hyperterminal, Advanced Serial Port Monitor, HyperACCESS, entre otros brindan la posibilidad de visualizar los datos en diferentes formatos. 


\section{Referencias}

Abhishek, Y. (2009). Digital Communication. Laxmi Publications Pvt. Ltd.

Artés Rodríguez, A., Pérez González, F., Cid Sueiro, J., López Valcarce, R., Mosquera Nartallo, C., \& Pérez Cruz, F. (2012). Comunicaciones Digitales. (DERECHOS RESERVADOS Los autores) Recuperado el 26 de Marzo de 2014, de http://www.tsc.uc3m.es/ antonio/libro_comunicaciones/El_libro_ files/comdig_artes_perez.pdf

Balcells, J., Autonell, J., Barra, V., Joan, B., Fornieles, F., García, B., \& Ros, J. (2011). Enlaces físicos. En Eficiencia en el Uso de la Enregía Eléctrica (págs. 222 - 225). Marcombo.

Bateman, A. (2003). Comunicaciones Digitales: diseño para el mundo real. Marcombo, S.A.

Beck, K. (1999). Extreme Programming Explained: Embrace Change. Addison Wesley.

Chitode, J. (2002). Digital Communications. Technical Publications Pune.

Coimbra, E. (2010). Comunicaciones Digitales.

Cortizo Pérez, J. C., Expósito Gil, D., \& Ruiz Leyva, M. (2002). ¿Cuándo y dónde usar la XP? En eXtreme Programming (págs. 13 - 14).

Inc., M. (1994). Discrete-Time Signal Trajectory Scope. Recuperado el 19 de Marzo de 2013, de http://www.mathworks.es/es/help/comm/ref/ discretetimesignaltrajectoryscope.html

Luque Rodríguez, J., \& Clavijo Suero, S. (1995). Modulación de Señales Digitales. Sevilla: Universidad de Sevilla, Departamento de Tecnología Electrónica.

Maral, G., \& Bousquet, M. (2010). Satellite Communications Systems: Systems, Techniques and Technology. Wiley.

MathWorks, I. (1994). CPFSK Modulator Baseband. Recuperado el 21 de Marzo de 2014, de http://www.mathworks.es/es/help/comm/ref/ cpfskmodulatorbaseband.html

MathWorks, I. (1994). Discrete-Time Eye Diagram Scope. Recuperado el 19 de Marzo de 2013, de http://www.mathworks.es/es/help/comm/ref/ discretetimeeyediagramscope.html

MathWorks, I. (1994). QPSK Modulator Baseband. Recuperado el 21 de Marzo de 
2014, de http://www.mathworks.es/es/help/comm/ref/qpskmodulatorbaseband.html

Muñoz Hidalgo, J. A., \& Zemanate Zuñiga, J. C. (2014). Análisis del Desempeño de un Sistema de Comunicaciones con Modulación 16/64 QAM Basado en Hardware Reconfigurable. Universidad del Cauca, Popayán.

Packard, H. (1997). Digital Modulation in Communications Systems - An Introduction. U.S.A: Hewlett-Packard Company.

Pérez, B. (2003). GXP - Adaptación y Aplicación de eXtreme Programming. Universidad de la República, Montevideo, Uruguay.

Prasad, K. V. (2003). Principles of Digital Communication Systems and Computer Networks. Charles River Media.

Quintero, V., \& Jojoa, P. (2013). Diseño e implementación de un prototipo de comunicación de datos basado en hardware reconfigurable Fase 1. Universidad del Cauca, Popayán.

Rondon, J., \& Sandoval, C. (2010). Diseño de un co-laboratorio remoto basado en programación modular de dispositivos VHDL aplicado a telecomunicaciones. Revista de la Facultad de Ingeniería Universidad Central de Venezuela, 25(2), 7-12.

The MathWorks, I. (1994). Discrete-Time Scatter Plot Scope. Recuperado el 19 de Marzo de 2013, de http://www.mathworks.es/es/help/comm/ref/ discretetimescatterplotscope.html

The MathWorks, I. (1994). Discrete-Time Signal Trajectory Scope. Recuperado el 04 de Febrero de 2014, de http://www.mathworks.com/help/comm/ref/ discretetimesignaltrajectoryscope.html

The MathWorks, I. (1994). GUI de MATLAB. Recuperado el 10 de Febrero de 2014, de http://www.mathworks.com/discovery/matlab-gui.html

Tung Lee, R. C., Chiu, M.-C., \& Lin, J.-S. (2007). Communications Engineering: Essentials for Computer Scientists and Electrical Engineers. John Wiley \& Sons Inc.

Wyglinski, A. M. (2013). Digital Communication Systems Engineering with Software-Defined Radio. Artech House.

XILINX. (2010). Spartan-3A FPGA Family. 19 Agosto.

XILINX. (s.f.). XILINX, All Porgrammable. Recuperado el 10 de Febrero de 2014, de http://www.xilinx.com/training/fpga/fpga-field-programmable-gate-array.htm

XILINX. (s.f.). XILINX, All Programmable. Recuperado el 10 de Febrero de 2014, de http://www.xilinx.com/tools/sysgen.htm 